\title{
COMIC STRIPS ET PAPIER GLACÉ. RÉTRO-RÉFLEXIVITÉ ET PSEUDO-SÉRIALITÉ DANS LitTLE TOMmy LOST
}

\author{
Benoît Crucifix ${ }^{1}$
}

\begin{abstract}
Cet article examine la place du comic strip au sein du roman graphique contemporain à travers l'analyse de Little Tommy Lost (2013) de l'auteur américain Cole Closser. La particularité de cette œuvre tient en ce qu'elle se propose comme un pastiche rigoureux de divers comic strips des années 1920 et 1930, allant jusqu'à se faire passer pour une collection d'anciens strips oubliés puis redécouverts. Little Tommy Lost creuse donc une vague rétro-réflexive qui interroge la façon dont la bande dessinée peut remédier ses propres identités culturelles. Il s'agira de cerner les enjeux stylistiques, sériels, narratifs et culturels d'une telle remédiation rétro-réflexive.
\end{abstract}

Au sein du catalogue de Koyama Press, Little Tommy Lost a de quoi détonner. La petite maison d'édition canadienne a été un véritable creuset de la bande dessinée alternative, offrant un espace de publication à de jeunes auteur-e-s désormais incontournables comme Eleanor Davis, Michael Deforge, Aidan Koch, ou Patrick Kyle. Influencé par le zine, l'art contemporain et l'illustration, tout en maintenant un rapport décomplexé à la culture populaire, le catalogue de Koyama Press reflète une tendance pointue de la bande dessinée contemporaine, que certains regroupent

1 Benoît Crucifix est aspirant FNRS à l'Université de Liège et à l'Université catholique de Louvain. Il est membre du groupe ACME (www.acme.ulg.ac.be).

Recherches en communication, $n^{\circ} 46$ - Article publié le 24/04/2018 
sous le terme d'art comics (Molotiu, 2017). Si les styles sont hétérogènes, le catalogue n'apparaît nullement nostalgique, avec peu de références aux traditions classiques de la bande dessinée américaine. Il s'agit donc très clairement, chez Koyama Press, de proposer du nouveau, et non de reformuler de l'ancien, dans une logique plurielle de distinctions ${ }^{2}$. Dans un tel contexte, les livres de Cole Closser, Little Tommy Lost (2013) et Black Rat (2015), font contraste et pourraient étonner par leur aspect graphique désuet, leur esthétique « rétro » faisant ouvertement référence à l'histoire de la bande dessinée. L'enjeu de Little Tommy Lost, qui sera au cœur de cet article, est précisément ce décalage anachronique, ce " faire semblant » d'un objet éminemment contemporain, mais qui joue à se présenter comme la résurgence d'un objet du passé.

Little Tommy Lost se lit en effet comme un hommage explicite à l' « âge d'or » du comic strip nord-américain et invite à une réflexion sur les manières dont le passé de la bande dessinée refait surface aujourd'hui, à la croisée du roman graphique et d'une pratique intensifiée de la réédition qui, selon Jan Baetens et Hugo Frey (2015), donne lieu à une véritable "rétromania » (Reynolds, 2011) dans le champ de la bande dessinée. Des figures canoniques comme Chris Ware, Seth, Art Spiegelman, ou Kim Deitch sont autant d'exemples d'auteurs contemporains qui sont aussi historiens de « leur » médium, et dont l'obsession pour le passé de la bande dessinée oriente fortement leurs propres bandes dessinées (Gardner, 2012, pp. 149-179; Jenkins, 2013).

L'objet qu'il s'agit d'étudier ici occupe cependant une place distincte par la simulation auquel l'auteur se prête, rendant l'ouvrage très ambivalent puisque Cole Closser nous confronte à une double forme médiatique : nous tenons dans nos mains un graphic novel, relié, souple, imprimé sur un épais papier glacé, flambant neuf, et en même temps le livre se fait sciemment passer pour une collection de vieux comic strips oubliés et tout juste redécouverts. Closser articule ainsi une certaine tension entre la pérennité matérielle supposée par sa matérialité et la qualité éphémère de la presse, vivier des comic strips auxquels il rend

2 On retrouve là des dynamiques similaires à celles d'un éditeur comme PictureBox (Méon, 2014). 
hommage. Alors que le comic strip contemporain - qu'on pensait voir mourir avec le déclin du journal papier - retrouve un nouvel élan sous la forme du webcomic, Closser propose quant à lui une « remédiation » (Bolter \& Grusin, 1999) du comic strip via le roman graphique tout à fait en lien avec les hybridations contemporaines entre graphic novel et rééditions patrimoniales ${ }^{3}$.

\section{Remédiation et rétro-réflexivité}

Appliquer le concept de remediation à un tel objet ne relève pas de l'évidence et nous pose d'emblée face à deux enjeux théoriques : d'une part, le caractère « intramédial » (Rajewsky, 2005, p. 46) de l'ouvrage de Cole Closser, et d'autre part, l'application problématique du concept à la bande dessinée. A priori, Little Tommy Lost ne relèverait en effet pas tellement d'une pratique intermédiale, mais plutôt d'une forme d'intertextualité à l'intérieur des frontières de son propre médium. Si Bolter et Grusin ne manquent pas de pointer du doigt ces pratiques « qui ne violent pas la présumée sainteté du médium » (1999, p. 49 ; nous traduisons) comme une forme interne de remédiation, le concept porte davantage sur " la représentation d'un médium au sein d'un autre » (p. 45), et donc sur les processus de remédiation d'un (ancien) médium par un autre (nouveau) médium. Pourtant, à condition de se garder des tendances du concept à niveler les spécificités matérielles des phénomènes médiatiques (Rajewsky, 2005, p. 64) et à linéariser les processus historiques (Baetens, 2009), le concept de remédiation peut toutefois offrir un éclairage intéressant sur les dynamiques du néo et du rétro, en ce qu'il souligne davantage le facteur temporel et matériel que les théories de l'intertextualité. Plutôt que de se décliner en relations intertextuelles (ou « hypertextuelles » selon Genette, 1982), le concept de remédiation peut permettre d'envisager la manière dont un médium recycle sa propre histoire d'une façon qui intègre ses identités multiples.

3 J'opte pour la traduction de remediation par « remédiation » à la suite de Jan Baetens (2009). 
C'est notamment ce que propose Brett Camper (2009) à travers le concept de « retro-reflexivity» (soit « rétro-réflexivité»), utilisé pour décrire une tendance dans le jeu vidéo qu'illustre La-Mulana, un jeu en 8-bit qui, bien que développé avec les technologies les plus avancées, a tous les aspects d'un jeu de console MSX des années 1980. Constatant le rôle que pouvait encore jouer des conventions obsolètes au sein de l'industrie du jeu vidéo dans la réflexion sur l'histoire du médium, Camper avance une redéfinition du concept de Bolter et Grusin, où

la remédiation se situe également au niveau "local" : au fur et à mesure de l'évolution d'un médium, ses étapes précédentes commencent à être remédiées en son sein. Le souci de légitimation ou de réalisme s'efface et la remédiation sort ainsi du plan de secours pour devenir un choix stylistique recherché, une tactique pour invoquer et réinterpréter le passé du médium, un vecteur privilégié pour l'hommage, la parodie, ou le revival de genres. C'est là que la remédiation se confronte au rétro (Camper, 2009, p. 186 ; nous traduisons).

Ce même lien organique entre technologie, genre et esthétique, ainsi que l'identification de ces aspects comme obsolètes, est également structurant dans une œuvre comme Little Tommy Lost qui peut être lue comme un pendant, en bande dessinée, de ce type de créations rétro-réflexives.

Néanmoins, la bande dessinée est moins structurée par l'innovation technologique et l'obsolescence correspondante de leur technicité, comme c'est le cas dans le jeu vidéo. Un tel phénomène pose davantage problème à la double logique de la remédiation avancée par Bolter et Grusin (1999, pp. 3-15) qui présentent le changement médiatique comme une perpétuelle oscillation entre immediacy et hypermediacy, transparence et opacité de la médiation. Privilégiant la trace et l'ellipse, la bande dessinée apparaît comme une forme culturelle toujours déjà un peu perdante sur le terrain de l'immédiateté de sa représentation et de l'efficacité de sa narration (Gardner, 2012). Elle reste alors un peu difficile à placer dans une histoire linéaire de la remédiation qui 
aurait très vite rendu la bande dessinée obsolète. Au contraire, la bande dessinée reste une forme fleurissante au sein de l'écologie médiatique du $21^{\mathrm{e}}$ siècle, une situation spécifique habilement cernée par Thierry Smolderen :

Alors qu'un crayon et du papier suffisent à produire une bande dessinée, la forme se porte comme un charme à l'âge du cinéma, de la radio, de la télévision, de l'informatique et d'Internet. Il me semble que la robustesse de ce médium rustique représente, au $20^{\mathrm{e}}$ siècle, sa caractéristique historique la plus étonnante

(Smolderen, 2012, p. 71).

Dans le même temps, l'histoire de la bande dessinée est inséparable de ses supports matériels et de ses moyens de reproduction. Il s'agit, comme le propose Smolderen dans Naissances de la bande dessinée (2009), de l'envisager suivant ses multiples transformations, en accord avec les changements de son écologie médiatique, plutôt que dans une perspective téléologique d' ' évolution » de la forme. Ces multiples naissances ne sont pas sans évoquer la généalogie des médias telle qu'elle a été théorisée par André Gaudreault et Philippe Marion (2012) dans leurs divers travaux. Dans un récent essai, Marion propose ainsi d'envisager la bande dessinée suivant ses identités plurielles et changeantes, dans une perspective diachronique où les enjeux d'institutionnalisation et d'appellation ont tout leur rôle à jouer. L'appellation est en effet un point nodal en ce qu'elle peut être " révélatrice de la manière dont le média organise les séries culturelles qui toujours le traversent et qu'il fédère au fil de son histoire » (Marion, 2016, p. 49).

La pertinence d'une telle approche est très clairement mise en valeur par les débats en Amérique du Nord autour du graphic novel, où l'appellation est véritablement perçue comme une catégorie littéraire en rupture avec la tradition des comics. Christopher Pizzino, dans Arresting Development (2016), montre ainsi la façon dont le discours médiatique cale l'histoire de la bande dessinée sur la trame téléologique du Bildungsroman, 
dans laquelle le roman graphique ferait figure de maturité. Or, si suivant Baetens \& Frey (2015), le roman graphique n'est pas seulement un support ou un «genre », mais un médium distinct, celui-ci est tout sauf le résultat d'un développement unilinéaire ou d'une simple « maturation » de la bande dessinée. Les deux auteurs montrent d'ailleurs que « la rupture (même symbolique) établie par le roman graphique a provoqué une grande fascination pour l'histoire du comic book» (Baetens \& Frey, 2015, p. 6 ; nous traduisons). En proie à une véritable « rétromania », qui s'exprime notamment par le volume de rééditions d'anciennes bandes dessinées, le graphic novel travaille aussi à une remédiation de sa propre histoire et des identités plurielles de la bande dessinée, explorant en profondeur une veine rétro-réflexive. À ce moment crucial où se rejoue l'identité de la bande dessinée, Little Tommy Lost de Cole Closser propose une forme de remédiation locale de l'histoire de la bande dessinée particulièrement attentive à sa matérialité, mais aussi à sa mémoire culturelle. Cette forme de remédiation désigne alors le mouvement de résurgence et de réinvention que d'" anciennes » identités du médium peuvent prendre dans un présent du médium traversé de multiples temporalités.

\section{Retour du comic strip en roman graphique}

Le caractère rétro-réflexif de Little Tommy Lost et le parallèle avec un jeu vidéo comme La-Mulana réside dans un jeu de « faire semblant » qui s'établit avec le lecteur et l'usager : tout en sachant très bien qu'il s'agit d'un produit contemporain, on est confronté à un ouvrage qui mobilise toutes les conventions d'une bande dessinée anachronique. C'est comme si Little Tommy Lost était véritablement un artefact du passé, paru dans les journaux américains des années 1920. L'ouvrage raconte les aventures d'un jeune enfant égaré, Little Tommy, qui se retrouve interné dans un orphelinat, puis tient tête aux brutes de l'institution et essaye de partir à la recherche de ses parents. Avec ce substrat dickensien et sa trame narrative basée sur l'abus d'une victime innocente, le pathos émotionnel, et la polarisation morale des personnages, ce 
récit s'inscrit tout à fait dans le mélodrame qui faisait florès dans les comic strips des années 1920 et 1930.

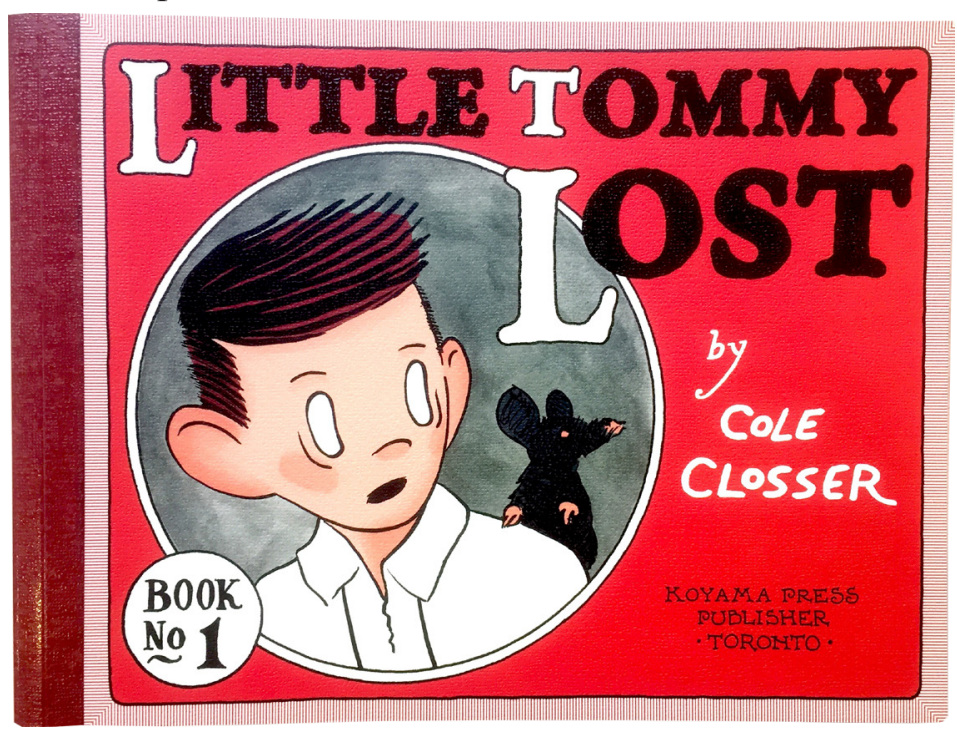

Figure 1. Cole Closser, Little Tommy Lost (Toronto : Koyama Press, 2013) (C) Closser 2013.

Little Tommy Lost, avec ses grands yeux vides et son rat comme animal de compagnie (Figure 1), est une réincarnation à peine voilée de Little Orphan Annie, le strip de Harold Gray qui débuta en 1924 dans les journaux américains, faisant le récit feuilletonnesque des péripéties d'une orpheline déterminée et courageuse, mais sans cesse confrontée aux aléas d'une société corrompue. Little Tommy Lost, cependant, ne se présente pas comme une réécriture de Little Orphan Annie, auxquels les strips de Gray, conversateurs et anticommunistes, auraient pourtant pu bien se prêter ${ }^{4}$. Une telle réécriture aurait souligné une distance historique avec l'original que Closser vise justement à confondre de façon ludique, puisque Little Tommy Lost ne doit pas tellement

4 Il faut ainsi noter les convictions républicaines de Harold Gray, qui a souvent tenté d'intervenir dans les débats politiques de son temps à travers Little Orphan Annie ; ce qui n'empêche pas de faire une lecture plus progressiste de la série, qui met en scène un personnage féminin doté d'une agency considérable sur sa propre situation (Wojcik, 2017). 
se lire comme notre contemporain, mais plutôt comme celui de Gray.

Outre Little Orphan Annie, Closser introduit une palette de références que tout amateur des comic strips du début vingtième reconnaîtra, et une grande partie du plaisir consiste ainsi en une lecture encyclopédique qui va répertorier les différentes allusions faites à l'histoire de la bande dessinée. On y retrouvera les déambulations automnales des pages dominicales de Gasoline Alley $^{5}$, les laids vilains de Dick Tracy ${ }^{6}$, le réveil au tomber du lit de Little Nemo in Slumberland ${ }^{7}$, les uppercuts et scènes de bagarre $\mathrm{du}$ Thimble Theatre ${ }^{8}$, les distractions enfantines de garnements comme Little Jimmy ${ }^{9}$ ou Naughty Pete ${ }^{10}$. Toutes ces références sont uniformisées par le trait de Closser qui reste homogène, et parfois un peu maladroit dans la mesure où le jeune artiste n'a pas la virtuosité graphique des « maîtres » qu'il pastiche.

Par cette imitation très poussée mais homogène de différents newspaper strips, Closser s'inscrit dans une approche rétroréflexive particulière, distincte d'autres approches manifestant un même hommage à cette période de l'histoire de la bande dessinée. Olivier Schrauwen, par exemple, livre avec My Boy (2006) un pastiche des œuvres de Winsor McCay, mais réadapté à un autre contexte et introduisant une ironie qui contraste avec le style emprunté (Meesters, 2010). Un autre exemple serait celui de Tony Millionaire, auteur de Maakies, comic strip publié de 1994 à 2016 dans la presse alternative ${ }^{11}$, et dont le style graphique désuet est très inspiré par une bande dessinée des premiers temps, contrastant là encore avec l'usage transgressif d'un humour cru, cynique, plus proche de l'underground. Une telle dimension ironique est complètement absente chez Cole Closser, chez qui le fairesemblant est capital, ce qui ramène Little Tommy Lost plus près de

5 Débuté en 1918 par Frank King dans le Chicago Tribune.

6 Débuté en 1931 par Chester Gould dans le Detroit Mirror.

7 Débuté en 1905 par Winsor McCay dans le New York Herald.

8 Débuté en 1919 par E. C. Segar dans le New York Evening Journal.

9 Débuté en 1904 par James Swinnerton dans le New York Journal.

10 Dessiné par Charles Forbell et publié en 1913 dans le New York Herald.

11 Certaines parties de Maakies ont été rassemblées en livre chez Fantagraphics, voir par exemple Millionaire (2000). 
la tradition du pastiche et de la rétro-réflexivité vidéoludique d'un La-Mulana. Au-delà du genre mélodramatique et d'un caractère encyclopédique, Closser donne en effet surtout à croire que les strips qu'il propose sont parus en journaux, sur un mode sériel. Il ne se réapproprie donc pas des textes ou un genre désincarné, mais propose plutôt une remédiation des formes matérielles de circulation et de transmission du comic strip, soulignant ainsi les dynamiques de la mémoire et de l'oubli.

\section{Pseudo-sérialité}

Il est bien nécessaire de souligner le « faux-semblant ${ }^{12}$ » de la démarche de Cole Closser, qui adopte le format " littéraire » du roman graphique, avec notamment une structure en chapitres. Relativement mince, totalisant 72 pages, Little Tommy Lost est un roman graphique dont le régime sériel est ambigu : l'ouvrage est présenté comme un premier volume, dont aucune suite ne semble prévue pour le moment. De fait, il s'agit donc d'un one-shot qui laisse son récit en suspens. En outre, le livre n'a pas connu de sérialisation au préalable, comme c'est pourtant souvent le cas dans le domaine du roman graphique. Cole Closser évite donc complètement la parution en série, mais réintroduit au sein de Little Tommy Lost tout l' « imaginaire sériel » (Letourneux, 2016) lié au comic strip. Ce faisant, Closser met à l'œuvre ce qu'on pourrait nommer une forme de pseudo-sérialité.

Closser segmente en effet son récit suivant une sérialité typique des comic strips des années 1920 et 1930, durant lesquelles la bande dessinée a exploré une forme de sérialité « ouverte », dont l'issue est indéterminée et toujours relancée, qui se répandra plus tard aux autres médias comme la radio et la télévision, auxquels le comic strip aura du mal à tenir tête (Gardner, 2013, p. 248). Avec des titres phares comme Little Orphan Annie, mais aussi The Gumps de Sidney Smith ou Wash Tubbs de Roy Crane, ces deux décennies marquent un moment tout particulier pour le comic

12 Ce qui évoque le « comme si » utilisé par Rajewsky (2005) pour décrire les références intermédiales, qui doivent toujours se faire dans les contraintes de la matérialité du médium propre. 
strip qui se caractérise par cette exploration innovante d'une sérialité ouverte. Le caractère nécessairement inachevé de Little Tommy Lost, restant ouvert à une potentielle continuation, marque déjà cette imitation d'une sérialité ouverte. Mais cette forme de sérialité particulière au comic strip de l'entre-deux-guerres se retrouve aussi dans l'alternance entre dailies, simples bandes de trois à quatre cases en noir et blanc paraissant quotidiennement durant la semaine, et Sunday pages, volet dominical de pleine page et en couleur. Little Tommy Lost reprend cette distribution hebdomadaire en segmentant le récit en six dailies consécutifs suivis d'une demi-page en couleur.

Par cette segmentation pseudo-sérielle, c'est la narration même qui s'en trouve contaminée. Les daily strips se nourrissent du double effet de répétition et de développement propre à établir une sensation de continuité et de suspense, « comme si » il fallait entretenir l'attention du lecteur de jour en jour. Closser a même recours aux vignettes-textes qui étaient introduites en début de strip lors des épisodes les plus hauts en tension, afin de faire raccord avec les épisodes précédents - comme c'est par exemple fréquemment le cas dans The Gumps, emblématique de ce mode sériel. Les pages dominicales, quant à elles et à l'instar d'une série comme Gasoline Alley, reflètent une pause dans la narration feuilletonnesque, souvent utilisée pour présenter les rêves ou les cauchemars de Little Tommy. Closser y joue régulièrement avec la tabularité de la page, transgressant les frontières des cases de façon à rappeler les expérimentations de Winsor McCay et Frank King (Figure 2). Plusieurs de ces pseudo-Sunday pages sont également basées sur des fêtes nationales telles qu'Halloween et Thanksgiving, autre caractéristique courante du comic strip qui doublait ainsi l'effet de lecture ritualisée en l'associant au calendrier annuel. Closser récupère donc à la fois la lecture sérielle du récit feuilletonnesque pour les strips en noir et blanc, et la lecture ritualisée des Sunday pages en couleur. 


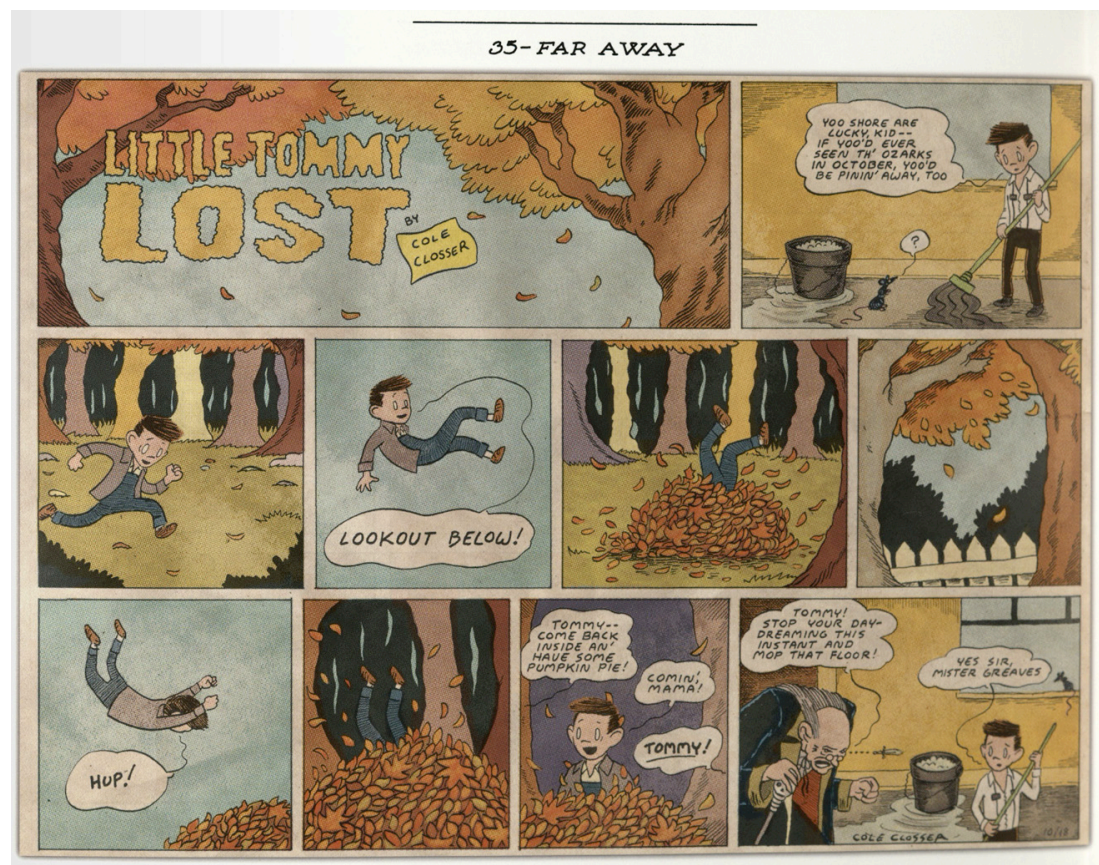

Figure 2. Cole Closser, Little Tommy Lost (Toronto : Koyama Press, 2013) (C) Closser 2013.

\section{Nostalgie analogique}

Tout le paradoxe de cette pseudo-sérialité est bien évidemment qu'elle est déconnectée autant du support du journal que des usages sociaux et culturels qui y sont liés : le rôle crucial des lecteurs, dont la participation et l'investissement dans le récit toujours en devenir sont capitaux (Gardner, 2012, pp. 46-47), y est totalement évacué. Ce retour au comic strip sous forme de roman graphique produit un paradoxe habilement relevé par Tony Millionaire dans l'éloge qu'il signe en quatrième de couverture de Little Tommy Lost : « [Closser] a créé une encyclopédie complète de newspaper comics. Ces bandes dessinées sont les plus rafraîchissantes que j'ai vues depuis que mon grand-père m'a montré sa collection de clippings de Roy Crane. Un travail fantastique qui, en plus, n'a pas besoin du journal » (cité dans Closser, 2013, quatrième de couverture). 
Un newspaper strip sans journal donc, mais une expérience de lecture que Millionaire relie à un résidu du passé dont la découverte même est rafraîchissante, illustrant le caractère novateur que peuvent prendre ces objets oubliés. Millionaire évoque en effet une collection de strips de Roy Crane, découpés dans le journal au fur et à mesure de leurs parutions et sans doute consignés dans un grand cahier : c'est sous la forme de scrapbooks que les lecteurs de comic strip auront souvent conservés leurs bandes dessinées favorites (Gardner, 2013, p. 149). Dans la continuité des scrapbooks du $19^{\mathrm{e}}$ siècle (voir Garvey, 2013), ces archives « sauvages » permettaient de combler un vide, de sélectionner et de préserver la mémoire d'un médium alors éphémère, pour lequel les démarches de réédition étaient isolées et fragmentaires. Rares exceptions, les livres publiés par Cupples \& Leon qui rééditait des comic strips au format carré, et dont Closser s'inspire directement pour sa couverture (Figure 1).

La stratégie qu'adopte Closser, peut-être justement pour palier au paradoxe de cette pseudo-sérialité, est justement de donner à Little Tommy Lost une apparence de scrapbook, par la façon dont chaque strip est isolé sur un fond blanc duquel il est détaché par une légère zone d'ombre, comme s'il y avait été collé. De plus, Closser prend grand soin à imiter la texture du papier de journal, ainsi que sa transparence et ses traces de dégradation (Figure 3). L'effet de transparence y est par exemple suggéré par l'inclusion d'une couche de texte qui se devine derrière les lignes et les traces dessinées du strip. Pour les pages « dominicales » en couleur, l'auteur utilise également un effet de trame qui rappelle les Ben Day dots utilisés pour l'impression en couleur des pages de journaux. Ces aspects de texture, de transparence, et de trame sont le résultat d'un minutieux travail numérique sur les dessins originaux, enrichis grâce au travail en multiples couches superposables caractéristique des logiciels numériques de traitement d'image. Closser y inclut également de nombreuses traces de dégradation, d'usure, de jaunissement, afin de rappeler la mauvaise qualité du papier sur lequel les comic strips étaient imprimés. 

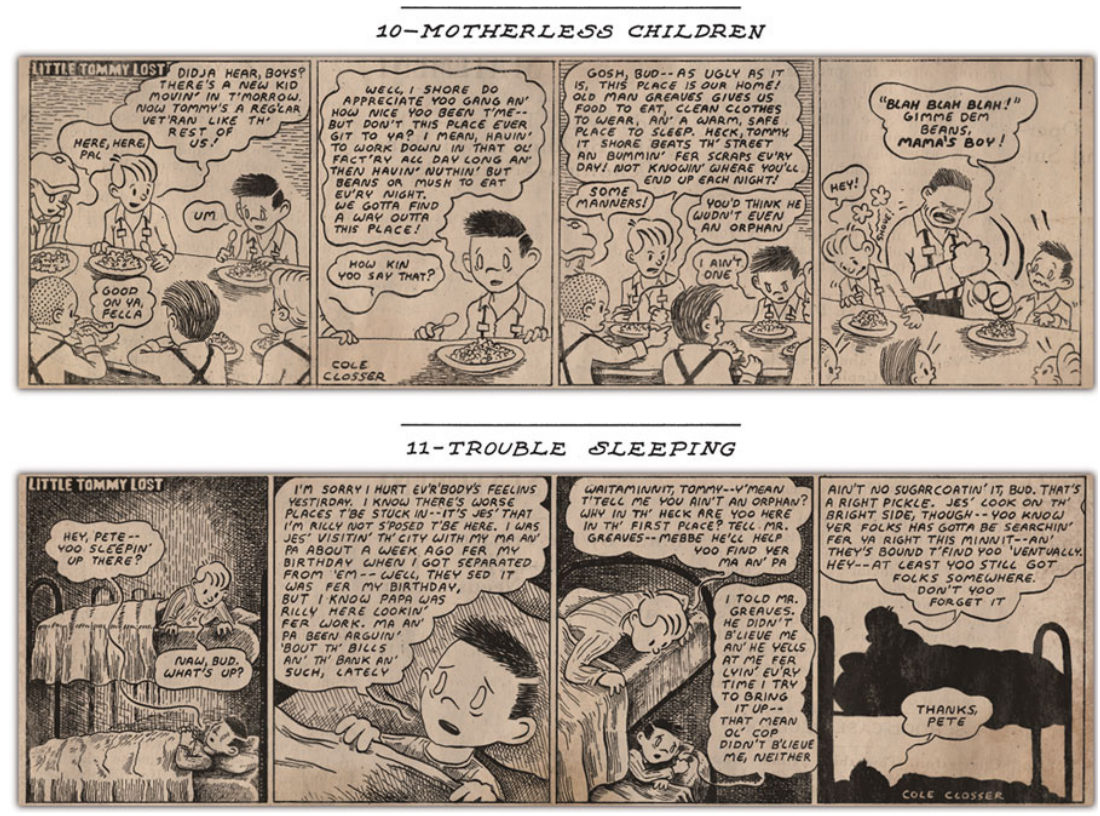

Figure 3. Cole Closser, Little Tommy Lost (Toronto : Koyama Press, 2013) (C) Closser 2013.

Ce genre de traitement numérique et de simulation d'un processus de dégradation manifestent une " nostalgie pour l'analogique » présente au sein des cultures numériques (Schrey, 2014). Parlant de vidéo et de cinéma, Laura Marks introduit le terme d' " analog nostalgia » pour décrire un « désir d'indexicalité » et une « affection rétrospective pour les "problèmes" de dégradation et de perte générationnelle que la vidéo analogique posait » (2002, p. 152). En se présentant comme un scrapbook oublié et redécouvert, dont les strips sont marqués par le passage du temps, Little Tommy Lost exprime une nostalgie pour ce support éphémère du journal, alors même que ses propres pages sont faites d'un épais papier glacé, plus apte à tenir l'épreuve du temps que les comic strips dont ils s'inspirent et qui tombent aujourd'hui en morceaux.

Ce contraste est aussi celui qu' on retrouve dans les nombreuses rééditions de « vieilles » bandes dessinées qui occupent depuis quelques années les étagères des librairies spécialisées. Il y aurait 
beaucoup à dire sur cette vague de rééditions, dont certaines ambiguïtés ont déjà été soulevées par Jan Baetens et Hugo Frey (2015), mais on se contentera de souligner qu'une certaine approche parmi ces rééditions participe d'une « nostalgie analogique » par un design qui met en valeur l'aspect jauni, vieux, désuet, abîmé du papier dégradé. L'approche est parfaitement illustrée par les travaux du designer Chip Kidd, dont la collaboration avec le photographe Geoff Spear met à chaque fois la bande dessinée (des Peanuts (Kidd 2003 ; Kidd, 2015) à Plastic Man (Spiegelman \& Kidd, 2001) et Batman (Kidd, 2008)) à l'honneur dans toute sa matérialité : planches originales, pages publiées sur du papier de mauvaise qualité, et objets de merchandising.

Une approche similaire est appliquée aux reproductions des planches reprises dans les anthologies de Dan Nadel, Art Out of Time (2006) et Art In Time (2010). Si Nadel est davantage soucieux de reproduire des exemples plus longs, là où Chip Kidd passe davantage par un travail de détail et de juxtaposition, chaque œuvre est photographiée dans sa matérialité propre (papier jaunissant, froissé, etc.), encadrée d'un fond blanc et imprimée sur papier glacé. En outre, avec un sous-titre tel que Unknown Comics Visionaries, de telles anthologies poursuivent un « objectif de redécouverte » (Méon, 2014, p. 84) qui tend à « élargir le canon de la bande dessinée en rendant à nouveau visibles et accessibles des auteurs “inconnus" ou "oubliés" "(Méon, 2014, p. 84). Bien que cet objectif témoigne d'un engagement de l'éditeur, cette approche est aussi plus globalement caractéristique des dynamiques de mémoire culturelle et du marché contemporain de la réédition.

En reprenant ces caractéristiques visuelles typiques des rééditions patrimoniales de bande dessinée, Little Tommy Lost tend à se faire passer pour l'une d'elles, comme une collection de comic strips d'aventure oubliés puis redécouverts. Le fait qu'un jeune auteur de bande dessinée dédie justement sa propre pratique à la création d'une fausse anthologie de comic strips oubliés, dans un geste rétro-réflexif rarement aussi poussé, montre aussi que cette « rétromania » n'est pas que le résultat de stratégies marketing de commercialisation nostalgique, mais qu'elles ont 
bel et bien un impact porteur sur de nouvelles générations. Closser connaît ces comic strips peut-être d'abord par leurs rééditions dont l'action médiatrice l'a poussé à rendre un hommage fidèle à cette bande dessinée du début du $20^{\mathrm{e}}$ siècle.

\section{En guise de conclusion}

La rétro-réflexivité de Little Tommy Lost met en valeur les dynamiques mémorielles de préservation et de recirculation de la bande dessinée, suggérant qu'une nostalgie pour le passé peut être le lieu d'une mémoire vive, plutôt qu'ossifiée, du médium. Toutefois, il faut noter que sa pseudo-sérialité, tout en rendant hommage à l'imaginaire sériel particulier aux comic strips des années 1920 et 1930, rompt aussi avec une longue tradition de la bande dessinée en ne faisant que simuler cette sérialisation, là où celle-ci ressurgit justement à l'ère du numérique avec le webcomic. On peut contraster l'approche rétro-réflexive de Closser par les propositions faites par l'auteur américain Frank Santoro (2016), qui invite à étudier l'histoire de la bande dessinée pour y trouver de nouvelles façons de s'approprier le contemporain : dans un geste plus proche de l'archéologie médiatique, il voit le contexte de publication des comic strips comme une inspiration pour naviguer le paratexte diffus d'Internet qui accueille aujourd'hui beaucoup de webcomics. Alors que Little Tommy Lost met en relief les pratiques d'archivage des comic strips (des scrapbooks aux rééditions contemporaines), cette rétro-réflexivité et cette nostalgie pour l'analogique fait pourtant l'impasse sur d'autres questions clés pour la bande dessinée en contexte numérique, à commencer notamment par la fragilité des webcomics auxquels Internet ne garantit pas une pérennité ${ }^{13}$.

13 À propos de mémoire culturelle numérique, voir De Kosnik (2016). 


\section{Références}

\section{Euvres citées}

Closser, C. (2013). Little Tommy Lost. Toronto : Koyama Press.

Closser, C. (2015). Black Rat. Toronto : Koyama Press.

Kidd, C. (Éd.). (2003). Peanuts. The Art of Charles M. Schulz. New York : Pantheon.

Kidd, C. (Éd.) (2008). Bat-Manga! The Secret History of Batman in Japan. New York: Pantheon.

Kidd, C. (Éd.). (2015). Only What's Necessary. Charles M. Schulz and the Art of Peanuts. New York : Harry N. Abrams.

Millionaire, T. (2000). Maakies. Seattle : Fantagraphics.

Nadel, D. (Éd.). (2006). Art Out of Time. Unknown Comics Visionaries, 1900-1969. New York : Harry N. Abrams.

Nadel, D. (Éd.). (2010). Art in Time. Unknown Comic Book Adventures, 1940-1980. New York : Harry N. Abrams.

Schrauwen, O. (2006). Mon fiston. Angoulême : L'An 2.

Spiegelman, A. \& Kidd, C. (2001). Jack Cole and Plastic Man. Forms Stretched to Their Limits. New York: Chronicle Books.

\section{Références secondaires}

Baetens, J. (2009). Études culturelles et analyse médiatique. Autour du concept de remédiation. Recherches en communication, 31, 79-91.

Baetens, J. \& Frey, H. (2015). The Graphic Novel. An Introduction. New York : Cambridge University Press.

Bolter, J. D. \& Grusin, R. (1999). Remediation. Understanding New Media. Cambridge : MIT Press.

Camper, B. (2009). Retro Reflexivity : La Mulana, an 8-Bit Period Piece. Dans B. Perron \& M. J. P. Wold (Éd.), The Video Game Theory Reader 2. (pp. 169-195). New York/Londres : Routledge.

De Kosnik, A. (2016). Rogue Archives. Digital Cultural Memory and Media Fandom. Cambridge : MIT Press.

Gardner, J. (2012). Projections. Comics and the History of Twenty-First-Century Storytelling. Palo Alto : Stanford University Press.

Gardner, J. (2013). A History of the Narrative Comic Strip. Dans D. Stein \& J.-N. Thon (Éd.), From Comic Strips to Graphic Novels. Contributions to the Theory and History of Graphic Narratives. (pp. 301-322). Berlin : De Gruyter.

Garvey, E. G. (2013). Writing with Scissors : American scrapbooks from the Civil War to the Harlem Renaissance. Oxford: Oxford University Press.

Gaudreault, A. \& Marion, P. (2012). La Fin du cinéma ? Un média en crise à l'ère du numérique. Paris : Armand Colin.

Genette, G. (1982). Palimpsestes. La Littérature au second degré. Paris : Seuil.

Jenkins, H. (2013). Archival, Ephemeral, and Residual : The Functions of Early Comics in Art Spiegelman's 'In the Shadow of No Towers'. Dans D. Stein \& J.-N. Thon (Éd.), From Comic Strips to Graphic Novels. Contributions to the Theory and History of Graphic Narratives. (pp. 301-322). Berlin : De Gruyter.

Letourneux, M. (2016). Introduction - la littérature au prisme des sérialités. Belphégor, 14, disponible à : https://belphegor.revues.org/794.

Marion, P. (2016). La bande dessinée et ses identités culturelles. Paysages et frontières. 
Dans M. Ahmed, S. Delneste \& J.-L. Tilleuil (Éd.), Le statut culturel de la bande dessinée. Ambiguités et évolutions. (pp. 39-52). Louvain-la-Neuve : Academia/ L'Harmattan.

Marks, L. U. (2002). Touch. Sensuous Theory and Multisensory Media. Minneapolis : Minnesota University Press.

Meesters, G. (2010). Les significations du style graphique : 'Mon fiston' d'Olivier Schrauwen et 'Faire semblant c'est mentir' de Dominique Goblet. Textyles, 36-37, 215-233.

Méon, J.-M. (2014). Tisser d'autres liens ? Pratiques éditoriales et discours critique de l'éditeur 'PictureBox' : Indépendance et champ de la bande dessinée. Dans C. Dony, T. Habrand \& G. Meesters (Éd.), La bande dessinée en dissidence : Alternative, indépendance, auto-édition. (pp. 79-92). Liège : Presses universitaires de Liège.

Molotiu, M. (2017). Art Comics. Dans F. Bramlett, R. T. Cook \& A. Meskin (Éd.), The Routledge Companion to Comics. (pp. 119-127). New York/Londres : Routledge.

Pizzino, C. (2016). Arresting Development. Comics at the Boundaries of Literature. Austin : Texas University Press.

Rajewsky, I. O. (2005). Intermediality, Intertextuality, and Remediation : A Literary Perspective on Intermediality. Intermédialités, 6, 43-64.

Reynolds, S. (2012). Rétromania : comment la culture pop recycle son passé pour s'inventer un futur. Marseille: Le mot et le reste.

Santoro, F. (2016). Herriman Riff. The Comics Journal, disponible à : http://www.tcj. com/herriman-riff/.

Schrey, D. (2014). Analogue Nostalgia and the Aesthetics of the Digital. Dans K. Niemeyer (Éd.), Media and Nostalgia : Yearning for the Past, Present and Future. (pp. 27-38). Londres : Palgrave.

Smolderen, T. (2009). Naissances de la bande dessinée de William Hogarth à Winsor McCay. Bruxelles : Les Impressions Nouvelles.

Smolderen, T. (2012). Histoire de la bande dessinée : questions de méthodologie. Dans É. Maigret \& M. Stefanelli (Éd.), La Bande dessinée : une médiaculture. (pp. 7190). Paris : Armand Colin.

Wojcik, P. R. (2017). Little Orphan Annie as Streetwalker. Dans M. Heimerman \& B. Tullis (Éd.), Picturing Childhood. Youth in Transnational Comics. (pp. 13-29). Austin : University of Texas Press.

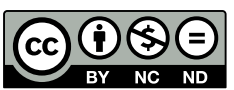

Publié sous la licence Creative Commons

«Attribution - Pas d'Utilisation Commerciale - Pas de Modification 4.0 International» (CC BY-NC-ND) 